\title{
The HERA Microscope
}

\section{Erdmann}

Universität Karlsruhe, Engesserstr. 7, D-76128 Karlsruhe

E-mail: Martin Erdmann@desy.de

ABSTRACT: The electron-proton collider HERA is a microscope, probing the structures of different objects made of quarks and gluons: proton, photon, mesons, and colour singlet exchange. Beyond this, in the searches for new particles and phenomena several events have been observed that are difficult to explain within the framework of the Standard Model. Examples are events at very high lepton scattering angles and events with an isolated muon and large missing transverse momentum.

$\mathrm{T}$ WO SURPRISING OBSERVATIONS have been made shortly after the commissioning of the electron-proton collider HERA at DESY in Hamburg 1992:

1. The number of constituents in the proton is extremely large. This measurement is explained by the gluons which confine the quarks to the proton. Since this observation, measurements on the gluon in hadronic matter were extended steadily:

a) the precision in the proton measurement was much improved,

b) for the first time the gluon was measured in the formation of hadronic matter using highly energetic photons, and

c) new types of measurements on the colour singlet exchange between two hadrons were carried through which imply that this state is dominated by gluons.

2. One event with an unexpected signature was found: it shows a highly energetic muon and a jet which are clearly separated from each other, and has in addition large missing transverse momentum. In total five muon events were found in the data taking up to 1997. Statistically one event is expected from calculations of the Standard Model. Other event signatures were observed which also show larger event yields than expected in the standard model.

To investigate particle structures and the ex- ceptional events further with 10 times the data statistics taken so far, a major upgrade of the HERA accelerator and the experiments $\mathrm{H} 1$ and ZEUS was decided. The start-up year is 2001. The preparation of the upgrade is ongoing with high priority.

\section{Measurements}

In two aspects the HERA accelerator is a milestone in the research on hadronic matter using leptons:

- Before HERA, fixed targets of Hydrogen were probed with electron, or muon, or neutrino beams. For the first time at HERA, conventional electron and proton accelerations were combined in a storage ring providing electron-proton collisions. The collider technique increases the center of mass energy of the collision by a factor of 10 to $\sqrt{s_{e p}}=300 \mathrm{GeV}$ and therefore reaches a resolution power for structures of $1 / 1000$ of the proton size.

- For the first time all interaction processes to studying the structures of particles are exploited within one multi-purpose experiment.

All measurements on the structure of a particle are carried out by scattering processes involving the constituents of the particle. The signatures of such processes are highly energetic leptons, 
hadrons, or photons which are found at large angles in the detector.

- The most obvious measurement is the detection of the scattered lepton. Using this technique the most important result so far on the structure of the proton was obtained: in figure $\overline{1}$, a recent measurement of the electron-proton cross section is shown in the form of the structure function $F_{2}\left[i_{1}\right]_{1}$. $F_{2}$ gives a measure of the quark density in the proton and is shown as a function of the fractional momentum $x$ of the scattered quark in the proton. The exact meaning of $F_{2}$ and the observables will be explained in section ' 2 '. Here we can already understand the meaning of the measurement from its shape: while above $x=0.01$ fixed target experiments found a moderate quark density in the proton, the surprising observation of the HERA measurements was the large number of constituents that populate the proton at small $x \leq 0.01$.

- A further signature for scattering processes involving the constitutions of a particle is jet production. The measurement of jet cross sections at HERA gave for the first time information on the gluons which are created in the formation of hadronic matter from highly energetic photons (section $\left.\overline{3_{1}^{\prime}}\right)$.

Beyond these measurement techniques a large number of additional signatures are exploited that allow different aspects of particle physics to be measured. A few examples are mentioned here:

- The measurement of events with the electron scattered at large angle and 2 jets allows the strong coupling constant $\alpha_{s}$ and its scale dependence to be measured.

- The simultaneous detection of the scattered lepton and the scattered proton gives information on elastic scattering processes and on the colour singlet state which carries the momentum exchange. For the first time the HERA measurements showed that this state is dominated by gluons (section $\left.\overline{4}_{1}^{-1}\right)$.

- The simultaneous measurement of the scattered electron and a neutron instead of the

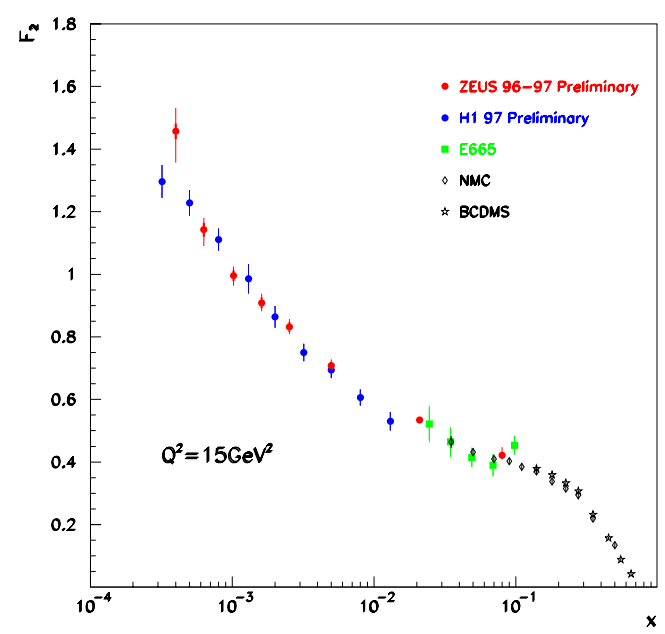

Figure 1: The proton structure function $F_{2}$ as a function of the fractional momentum $x$ of the scattered quark in the proton: only the HERA measurements show the large density of constitutions in the proton at small values of $x<10^{-2}$.

scattered proton gives information on the structure of the pion. The pion structure at small quark momenta $x=0.001$ is analysed at HERA for the first time.

- Measurements on the formation of vector mesons in elastic electron-proton scattering processes provide information on the structure of the mesons and give as well a highly sensitive measure of the gluon density in the proton.

- Detection of hadrons with charm or bottom quarks tests universality of the heavy quark interaction dynamics and gives information on their contribution to the structure of a particle.

- The signature of an isolated muon can be very important in the search for new particles and new phenomena (section

In the course of the past seven years many interesting results were obtained from the data analysis using the large number of measurement techniques. In this contribution, only the most important achievements in the measurements of particle structures are shown as well as the events which at least potentially hint for phenomena beyond the Standard Model. 


\section{The Structure of the Proton}

Since the discovery of constituents in the proton using electron-proton scattering 1969, measurements of the proton structure have been continuously improved and extended. The calculation of this proton structure from fundamental principles remains one of the major challenges to theory. However, the mechanisms how the constituents interact with other particles is well understood within the framework of Electro-Weak theory and Quantum Chromodynamics (QCD).

\subsection{Electro-Weak In- teraction Processes}

In deep inelastic electronproton scattering processes the electron is scattered off a quark from the proton. Electro-p Weak theory describes the electron-quark interaction by boson exchange: for neutral processes by photon or $\mathrm{Z}$

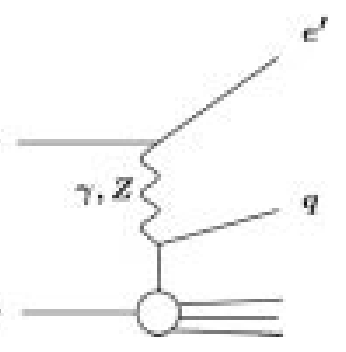

Figure 2: Deep inelastic electron-proton scattering with photon or $\mathrm{Z}$ boson exchange.

boson exchange, and for charged processes by $\mathrm{W}$ boson exchange (figures $\overline{2} 2, \overline{1}, \overline{1}, 1)$.

Example events are shown in figure 'in $_{n}^{\prime}$ Both events show the formation of a jet which indicates scattering of a constituent particle of the proton. In neutral exchange processes the scattered electron is found in the detector (figure $\overline{4}$ ). In the case of charge exchange

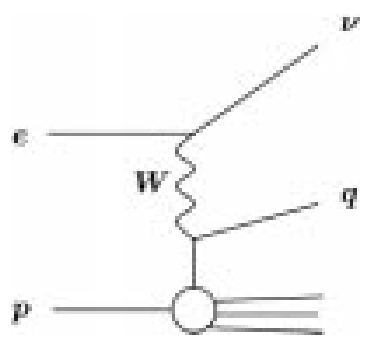

Figure 3: Deep inelastic electron-proton scattering with $\mathrm{W}$ exchange. processes the escaping neutrino is identified by missing transverse momentum (figure

The characteristics of such scattering processes are similar to the classical Rutherford experiment where the structure of atoms was investigated. The differential cross section for the electro-magnetic interaction of 2 pointlike particles is proportional to the interaction strength and to the inverse fourth power of the sine with

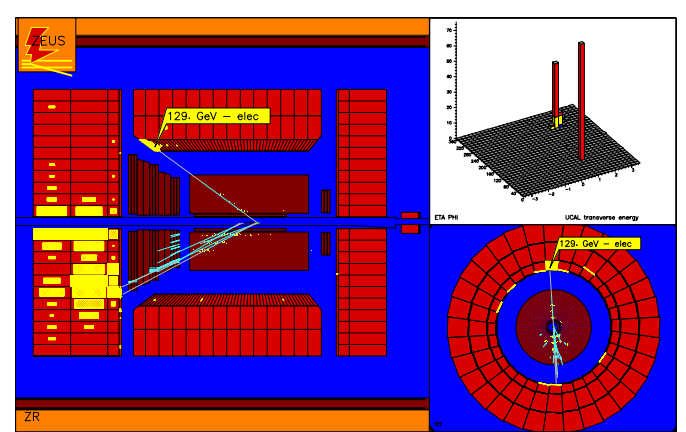

Figure 4: Deep inelastic electron-proton scattering with photon or $\mathrm{Z}$ exchange: the main display (left) gives the side view of the Zeus detector where the electron enters from the left and the proton from the right. The electron is visible at large scattering angle in the upper half. The jet pointing to the lower left direction reflects the scattered quark. The lower right part of the display gives the view along the beam line showing that the electron and jet are "back-to-back". The upper right histogram shows the distributed energies in the phase space of rapidity and azimuthal angle.

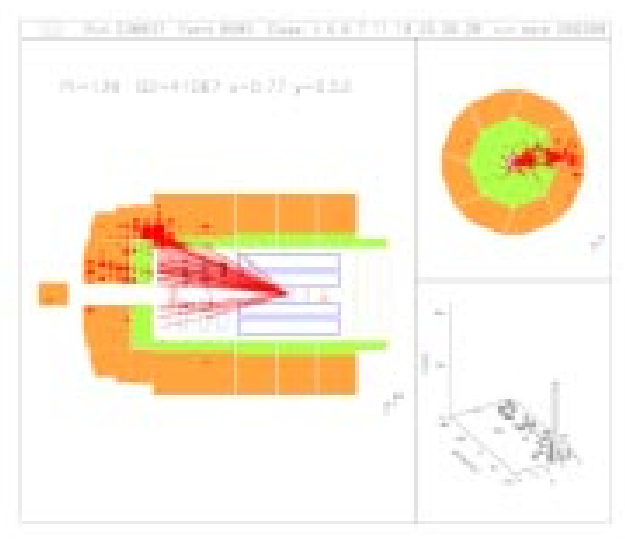

Figure 5: Deep inelastic electron-proton scattering with $\mathrm{W}$ exchange: The left display gives the side view of the $\mathrm{H} 1$ detector where the electron enters from the left and the proton from the right. The jet pointing to the upper left direction reflects the scattered quark. Neither in this view nor in the view along the beam line (right) the jet is balanced which indicates a highly energetic neutrino escaping the detector. The lower right histogram shows the distributed energies in the phase space of rapidity and azimuthal angle.

the argument being half of the scattering angle of the beam particle measured in the laboratory 
frame:

$$
\frac{\mathrm{d} \sigma}{\mathrm{d} \theta} \propto \alpha^{2} \frac{1}{\sin ^{4} \frac{\theta}{2}} .
$$

This calculation needs to be modified in several respects when investigating the proton structure in deep inelastic electron-proton scattering:

$$
\begin{aligned}
\frac{\mathrm{d}^{2} \sigma_{\gamma, Z}}{\mathrm{~d} Q^{2} \mathrm{~d} x} & \sim \alpha^{2} \frac{1}{Q^{4}} \frac{1}{x} \Phi_{\gamma, Z}\left(x, Q^{2}\right) \\
\frac{\mathrm{d}^{2} \sigma_{W}}{\mathrm{~d} Q^{2} \mathrm{~d} x} & \sim G_{F}^{2}\left(\frac{M_{W}^{2}}{M_{W}^{2}+Q^{2}}\right)^{2} \frac{1}{x} \Phi_{W}\left(x, Q^{2}\right)
\end{aligned}
$$

(a) The observable $\left(Q^{2}\right)^{2}$ is used instead of the $\sin ^{4}(\theta / 2)$ term. $Q^{2}$ is independent of the reference frame and provides a measure of the resolution power for structures of size $\lambda\left(\sqrt{Q^{2}} \propto \lambda^{-1}\right)$. It is calculated from $Q^{2} \equiv 4 E_{e} E_{e^{\prime}} \sin ^{2}(\theta / 2)$ where $E_{e}$ and $E_{e^{\prime}}$ denote the electron beam energy and the scattered electron energy respectively.

In the case of $\mathrm{W}$ exchange (equation $2.3 \mathrm{3})$, the distribution in the scattering angle is modified by the large boson mass $\left(M_{W}=\right.$ $80 \mathrm{GeV})$. This is also valid for the exchange of $\mathrm{Z}$ bosons $\left(M_{Z}=90 \mathrm{GeV}\right)$ where the effect is contained in $\Phi_{\gamma, Z}$.

(b) While the proton momentum is given by the accelerator, the proton structure implies that the momenta of the scattered quarks vary event by event. This quark momentum is determined relative to the proton momentum and is denoted by the dimensionless Bjorken variable $x$. The information on the proton structure is contained in the terms $\Phi_{W}$ and $\Phi_{\gamma, Z}$.

(c) The strength of the electro-magnetic interaction is given by $\alpha$. In the case of weak interactions, the strengths is relatively suppressed (for $\mathrm{W}$ exchange it is denoted by the Fermi-constant $G_{F}$, for $\mathrm{Z}$ exchange it is contained in $\left.\Phi_{\gamma, Z}\right)$.

(d) A characteristics of the neutral exchange processes is the interference of the photon and $\mathrm{Z}$ boson contributions which effectively gives a cross section enhancement for electronproton scattering and a deficit for positronproton scattering.
Leptons and quarks are fermions carrying spin $1 / 2$. Therefore two scattering components exist: if the spins add up to 0 , the distribution in the scattering angle $\theta^{*}$ in the center of mass system of the lepton and quark is uniform. If both spins add up to 1 , the scattering angles follow a distribution according to

$$
\cos ^{4} \frac{\theta^{*}}{2}=\left(1-\frac{Q^{2}}{s_{e p} x}\right)^{2}
$$

with $\sqrt{s_{e p}}$ being the lepton-proton center of mass energy. This scattering characteristics is contained in the "helicity weighted structure function terms" $\Phi_{W}$ and $\Phi_{\gamma, Z}$ together with the quark distributions of the proton:

The processes with charged $\mathrm{W}$ exchange involve neutrinos (left-handed helicity) and antineutrinos (right-handed helicity). The helicity rules of the Electro-Weak interactions imply that quarks and antiquarks of the proton are distinguished by the distributions in the scattering angle and from the charge of the incoming lepton:

$$
\begin{aligned}
& \Phi_{W}^{e^{-} p}\left(x, Q^{2}\right) \sim x u+\cos ^{4}\left(\frac{\theta^{*}}{2}\right) x \bar{d} \\
& \Phi_{W}^{e^{+} p}\left(x, Q^{2}\right) \sim x \bar{u}+\cos ^{4}\left(\frac{\theta^{*}}{2}\right) x d
\end{aligned}
$$

The $x u$ or $x d$ denote the probabilities of finding a $u$ or $d$ quark with fractional momentum $x$ in the proton. The bar-sign denotes the antiquarks. These probability distributions depend on $x$ and $Q^{2}$.

In photon exchange processes all quarks and antiquarks are probed such that

$$
\begin{aligned}
\Phi_{\gamma, Z}\left(x, Q^{2}\right) & \sim\left(1+\cos ^{4} \frac{\theta^{*}}{2}\right) \\
& \times\left(e_{u}^{2}(x u+x \bar{u})+e_{d}^{2}(x d+x \bar{d})\right) \\
& + \text { additional terms with } \gamma / Z
\end{aligned}
$$

Here $e$ denotes the fractional electric charge of the quarks which influence the strengths of the electro-magnetic processes.

The sum of the probability distributions of finding quarks in the proton, weighted by their squared electric charges, is conventionally written in terms of the structure function $F_{2}$ :

$$
F_{2}\left(x, Q^{2}\right)=e_{u}^{2}(x u+x \bar{u})+e_{d}^{2}(x d+x \bar{d})+\ldots
$$


At small values of $Q^{2}$ the cross section for leptonproton scattering is dominated by photon exchange. Here the structure function $F_{2}$ can be directly determined from the measurement of the double differential cross section (equations $\overline{2} .2$, (2.7)

$\frac{\mathrm{d}^{2} \sigma}{\mathrm{d} x \mathrm{~d} Q^{2}} \sim \alpha^{2} \frac{1}{Q^{4}} \frac{1}{x}\left(1+\cos ^{4} \frac{\theta^{*}}{2}\right) F_{2}\left(x, Q^{2}\right)$.

One example is shown in figure $\overline{1}_{1}^{1}$.

\subsection{Measurement of the Valence Quarks}

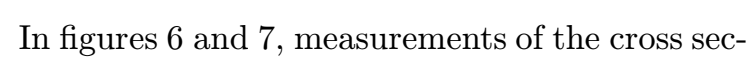
tions from positron-proton scattering are shown in terms of $\Phi_{W}$ and $\Phi_{\gamma, Z}$ (equations $\overline{2} .2,2 . \overline{3}$ ) [i] The data correspond to relatively large fractional momenta $x$ where the valence quarks of the proton are probed. The main trend is a linear dependence in $\cos ^{4}\left(\theta^{*} / 2\right)\left(\theta^{*}=\right.$ scattering angle in the electron-quark center of mass system) which is expected for the scattering of fermions with spin $1 / 2$ (equations $2.6,2.7 \overline{2}$ ).

For W exchange, two components of different magnitude are observed. The measured cross section extrapolated to $\cos ^{4}\left(\theta^{*} / 2\right)=0$ reflects the density of $\bar{u}$-quarks with an isotropic distribution of the scattering angle (dashed line, equation $2 . \overline{6}$ ). The contribution above the dashed line reflects the $d$ quarks which follow the expected distribution in the scattering angle (full curve). The curves result from a linear fit to the data. In this kinematic region, this simple fit follows closely the elaborate fits to many more data points which are shown in the following section. With increasing quark fractional momentum $x$ the contribution of the $\bar{u}$-quarks disappears while the $d$ valence quark contribution remains.

The measurements involving photon and $\mathrm{Z}$ exchange show a similar linear distribution, except for the data at high $x$ and small values of $\cos ^{4}\left(\theta^{*} / 2\right)$ where the negative photon-Z interference modifies the linear behavior. The size of this interference effect can be estimated from the difference between the extrapolated full curve and the data points at $x=0.25$. Except for these points, the bulk of the measured data shows the isotropic and the weighted components in the distribution of the scattering angle. The data

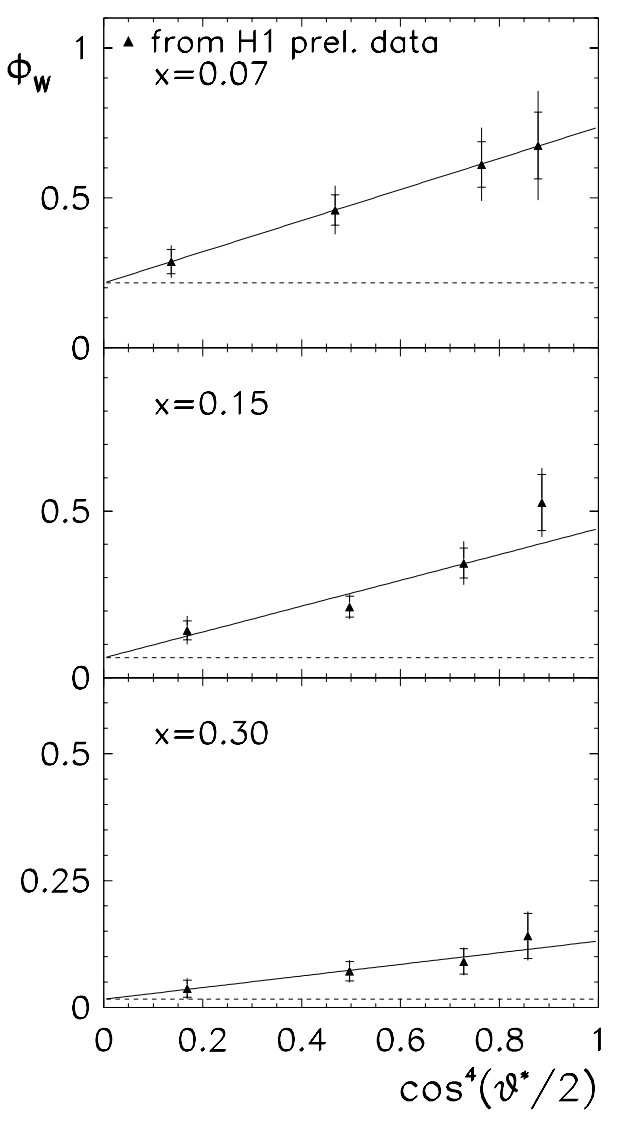

Figure 6: Helicity weighted structure function term $\Phi_{W}$ for processes with W boson exchange as a function of $\cos ^{4}\left(\theta^{*} / 2\right)$ where $\theta^{*}$ is the angle in the positron-quark center of mass system. Different regions of the fractional momenta $x$ of the scattered quarks are shown. The curves indicate the two components to the angular distribution which reflect the $\overline{\mathrm{u}}$ contribution (dashed line) and the $d$ valence quark contribution (above the dashed line).

are compatible with equal contributions of both components (equation 2.7 ) as indicated by the 1-parameter linear fit (full and dashed curves). Owing to the charge dependence of the electromagnetic interactions the contribution of $u$ quarks $\left(e_{u}^{2}=4 / 9\right)$ dominates the contribution of the $d$ quarks $\left(e_{d}^{2}=1 / 9\right)$. Therefore the cross section measurement extrapolated to $\cos ^{4}\left(\theta^{*} / 2\right)=$ 1 mainly reflects the total contribution of the $u$ quarks $(2 \times 4 / 9 x u)$.

The comparison of the $\Phi_{W}$ (figure $\overline{6}, \overline{1}$, domi- 


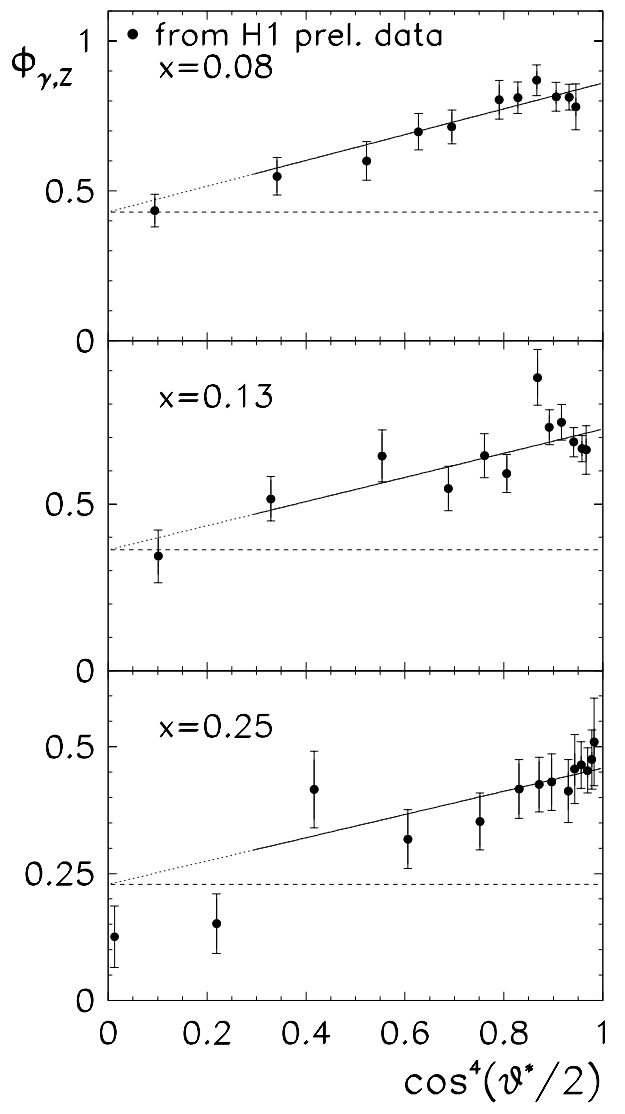

Figure 7: Helicity weighted structure function term $\Phi_{\gamma, Z}$ for processes with photon or Z boson exchange as a function of $\cos ^{4}\left(\theta^{*} / 2\right)$ where $\theta^{*}$ is the angle in the positron-quark center of mass system. Different regions of the fractional momenta $x$ of the scattered quarks are shown. The full and dashed curves indicate the two components to the angular distributions. At small values of $\cos ^{4}\left(\theta^{*} / 2\right)$ the cross section is reduced by the interference between the photon and $\mathrm{Z}$ contributions.

nantly $d$ quarks) and $\Phi_{\gamma Z}$ (figure $\overline{\bar{T}_{1}}$, dominantly $u$ quarks) measurements extrapolated to $\cos ^{4}\left(\theta^{*} / 2\right)=$ 1 shows a $u$ valence quark contribution in the proton that is about twice larger than the contribution of the $d$ valence quarks.

The errors in the measurements are determined by the statistics of the data. Precise statements on the valence quark distributions of the proton at the 10- fold increased resolution power in comparison to former fixed target experiments will be possible after the upgrade of the HERA accelerator and of the experiments $\mathrm{H} 1$ and ZEUS.

\subsection{Gluon Distribution from Scaling Vio- lations}

The measurement of the quark distributions using deep inelastic lepton-proton scattering is influenced by strong interaction phenomena. These phenomena make the interpretation of the measured cross sections more complicated, however, they indirectly allow the gluon distribution and the strong coupling constant $\alpha_{s}$ to be measured very precisely. Immediately before the electronquark scattering process occurs the quark may loose momentum by radiating a gluon. With increased resolution power $Q^{2}$ these radiation effects are better seen and therefore change the measured quark distributions. The effect is referred to as "scaling violations".

The interplay between these gluon radiation effects and the momentum distributions of the constituents of the proton is consistently described within the theory of Quantum Chromodynamics (QCD). The quarks which scatter off the electron are not only permanent constituents of the proton (valence quarks), but can also result from the splitting of a gluon into a quark-antiquark pair. The probabilities $x q, x g$ of finding a quark or a gluon in the proton are therefore interrelated in "QCD evolution equations":

$$
\begin{aligned}
\frac{\mathrm{d} x q}{\mathrm{~d} \ln Q^{2}} & =P_{q \rightarrow q g}(x) \oplus x q\left(x, Q^{2}\right) \\
& +P_{g \rightarrow q \bar{q}}(x) \oplus x g\left(x, Q^{2}\right) \\
\frac{\mathrm{d} x g}{\mathrm{~d} \ln Q^{2}} & =P_{q \rightarrow g q}(x) \oplus x q\left(x, Q^{2}\right) \\
& +P_{g \rightarrow g g}(x) \oplus x g\left(x, Q^{2}\right) .
\end{aligned}
$$

The splitting functions $P$ are predicted by QCD; they depend on the strong coupling constant $\alpha_{s}$. The $\oplus$ sign denotes an integration over all possible fractional momenta $x$.

In figure function $F_{2}$ are shown as a function of the resolution power $Q^{2}$ for different values of the measured fractional momentum $x$ of the quarks The open symbols seen in the lower left corner of the histogram are the measurements of the fixed target experiments. The closed symbols show the 
HERA measurements which cover, owing to the large center of mass energy, five orders of magnitude in $Q^{2}$ and $x$. Only in the central valence quark region around $x \sim 0.1$ the quark density in the proton is found to be independent of the resolution power $Q^{2}$ of the scattering process. At larger values of $x$ an increasing resolution does not show more constituents in the proton. Below $x=0.1$ the quark density increases with the resolution power. This behavior increases with smaller $x$ values down to $x \sim 0.001$.

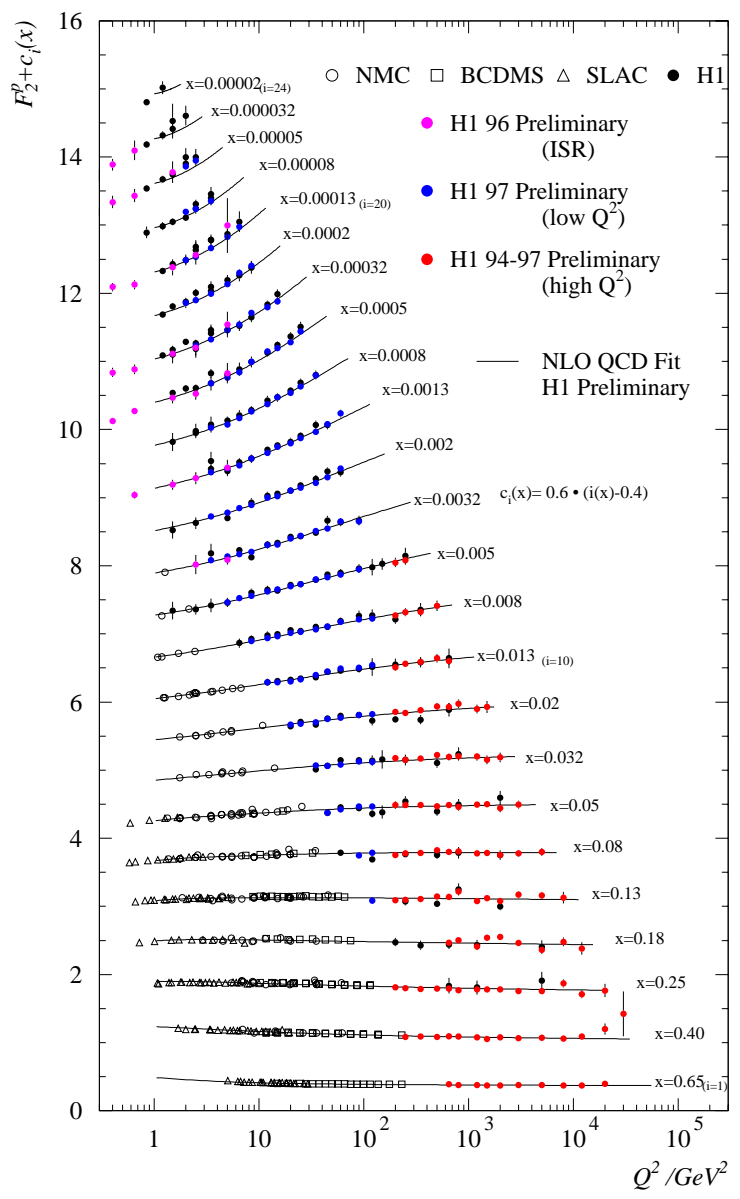

Figure 8: Proton structure function $F_{2}$ as a function of the resolution power $Q^{2}$ in different regions of the fraction momentum $x$ of the scattered quark. The curves are QCD fits to the data.

In figure $\overline{1}$, the curves reflect the result of a fit to the data using parameterizations for the quark and gluon distributions of the proton and determining the parameter values according to the QCD evolution equations ( $\overline{2} . \overline{1} 0,1, \overline{2} . \overline{1})$.

An example of the quark and gluon distri- butions of the proton is shown in figure as a function of the fractional momentum $x$ at fixed $Q^{2}=20 \mathrm{GeV}^{2}\left[\begin{array}{l}{[3]} \\ {\left[\beta_{1}\right.}\end{array}\right]$. At large values of $x$ the $u$ and $d$ valence quarks are found with high probability. The contribution of sea quarks, including strange and charm quarks, are found at small values of $x$. The gluon density in the proton is extremely large and has been reduced by a factor of 10 in the figure. This large number of gluons explains the high density of constituents that is seen in the proton structure function measurements at HERA (figure $\overline{1} \overline{1}$ ).

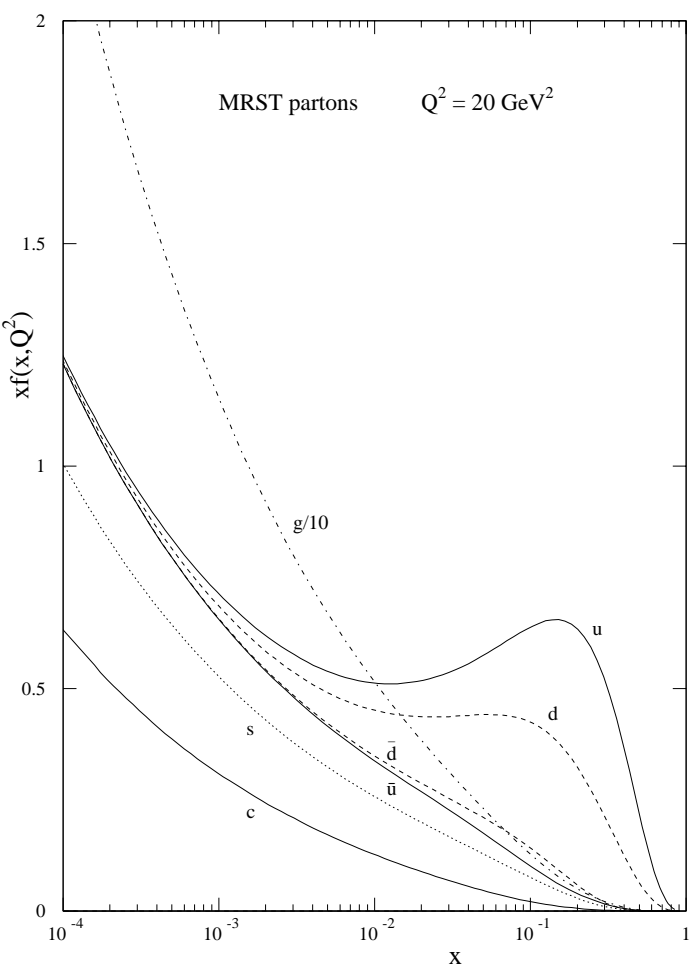

Figure 9: Parameterizations of the probabilities of finding quarks or gluons in the proton with fractional momenta $x$. The results follow from QCD fits to the structure function data.

\section{The Structure of the Photon}

The photon in electron-proton scattering does not only play the role of an exchange particle of the electro-magnetic interaction between electron and quark, but in itself constitutes an object to deepen our understanding of the formation of hadronic matter. 
The Heisenberg uncertainty relation allows energy and momentum conservation to be violated for a short time. The HERA beam electrons emit photons which for a short time can fluctuate into a quark-antiquark state $q \bar{q}$. These $q \bar{q}$ states of the photon are analysed in scattering processes with the quarks and gluons of the proton.

At the electron-positron accelerators in the 1980ies, such quark-antiquark states of the photon were already studied. The main aspects of the formation process are correctly predicted by Quantum Electrodynamics (QED) and Quantum Chromodynamics (QCD). The development of gluons in such $q \bar{q}$ states, which give the key to understanding the formation of a hadronic bound state, however, was unknown before HERA.

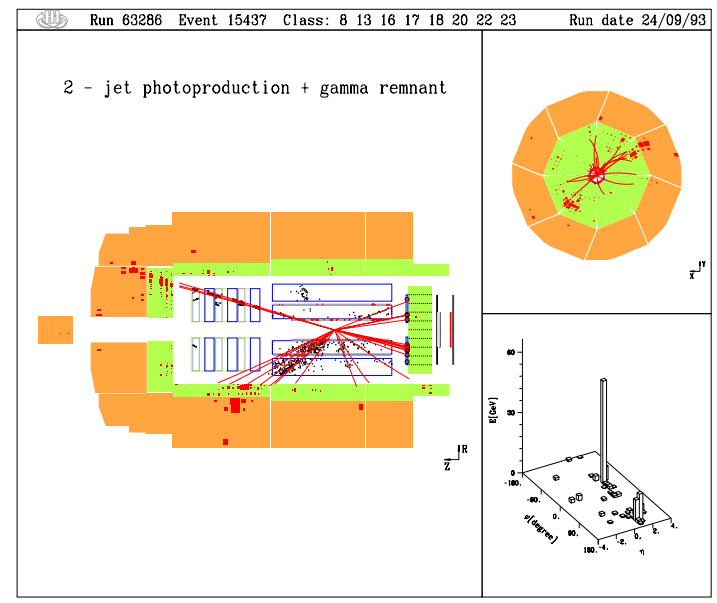

Figure 10: Di-jet production in photon-proton collisions: the main display (left) gives the side view of the $\mathrm{H} 1$ detector where the electron together with the photon enters from the left and the proton from the right. The jets pointing to the upper and lower left directions reflect the scattered quarks or gluons of the photon and proton. The particles pointing to the right side indicate that only a fraction of the photon momentum was involved in the jet production process. The upper right part of the display gives the view along the beam line showing that the two jets are "back-to-back". The lower right histogram shows the distributed energies in the phase space of rapidity and azimuthal angle.

\subsection{Strong Interaction Processes}

In scattering processes between the photons of the electron beam and the protons, events with two jets were observed where only a fraction of the photon momentum is involved in the jet production (figure $\overline{1}_{1}^{\prime} \overline{1}_{1}^{\prime}$ ). In such processes a quark or a gluon of the photon scatters off a quark or a gluon of the proton. Again this scattering process mainly follows the characteristics of Rutherford scattering, in this case with the gluon as exchange particle of the strong interactions (figure 1 lib). The differential cross section is
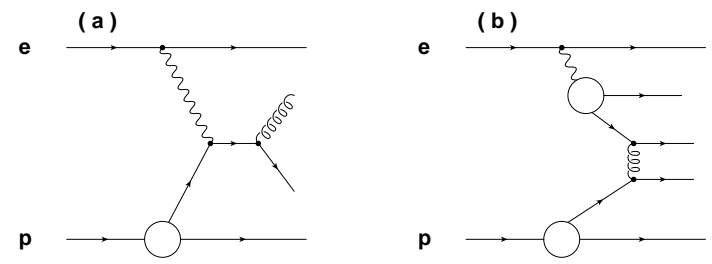

Figure 11: Mechanisms for di-jet production in photon-proton scattering: a) direct photon-proton interactions and b) interactions that involve the constituents of the photon and the proton.

predicted within QCD theory:

$$
\frac{\mathrm{d}^{3} \sigma_{\gamma p}}{\mathrm{~d} t \mathrm{~d} x_{\gamma} \mathrm{d} x_{p}} \sim \alpha_{s}^{2} \frac{1}{t^{2}} \frac{1}{x_{\gamma} x_{p}} \Phi_{g}\left(x_{\gamma}, x_{p}, t\right)
$$

The scattering angle $\theta^{*}$ in the center of mass system of the constituents is directly related to the observable

$$
t^{2} \propto\left(1-\cos \theta^{*}\right)^{2}=\sin ^{4}\left(\theta^{*} / 2\right) .
$$

The fractional momenta of the constituents of the photon and proton are denoted by $x_{\gamma}$ respectively $x_{p}$ and are calculated event-by-event from the jet configuration. The strength of the interaction is given by the strong coupling constant $\alpha_{s}$. Since scattering processes between quarks and gluons cannot be distinguished, the probabilities of finding a quark or gluon with the according fractional momenta were combined considering the enhanced interactions strength of the gluons (colour factor 9/4):

$$
\begin{aligned}
\Phi_{g} \sim & \left(1+\cos ^{4} \frac{\theta^{*}}{2}\right) \times \\
& \left(x_{\gamma} q_{\gamma}+\frac{9}{4} x_{\gamma} g_{\gamma}\right)\left(x_{p} q+\frac{9}{4} x_{p} g\right)
\end{aligned}
$$

In figure, $1 \overline{2}^{1}$ a measurement of the di-jet cross section is shown as a function of the cosine of the scattering angle $\theta^{*}[\overline{4}]$. The data strongly 
increase towards small angles, i.e. large $\cos \theta^{*}$. They are well described by QCD calculations (curves) which take into account scattering processes between quarks and gluons of the photon and the proton as well as direct interactions of the photon with the proton constituents (figure $\left.\overline{1}^{1} \overline{1}_{1}^{\prime}\right)$.

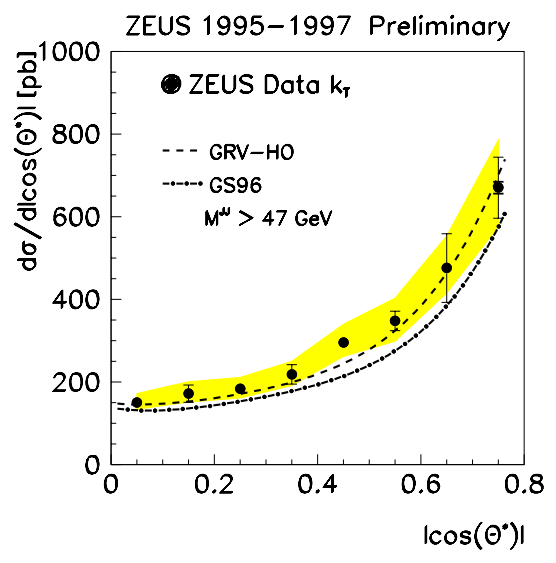

Figure 12: Di-jet cross section as a function of the cosine of the scattering angle in the center of mass system of the constituents coming from the photon and the proton. The curves represent QCD calculations.

\subsection{Gluon Formation in the Hadronization of Photons}

To measure the gluon contribution to the quarkantiquark state of a photon, the di-jet cross section was measured as a function of the fractional momentum $x_{\gamma}$ of the quarks or gluons from the photon. Except for the probability of finding a gluon in the photon $x_{\gamma} g_{\gamma}$ all components of equation $3 . \overline{3}$ are known: the quark distributions $x_{\gamma} q_{\gamma}$ of the photon have been measured at electronpositron accelerators, and the quark and gluon distributions of the proton are known from deep inelastic electron-proton scattering. Using the di-jet measurement, the gluon distribution of the photon was determined at HERA for the first time. A recent measurement of the combined quark and gluon density distribution of the photon is shown in figure $13_{1}^{1}\left[\begin{array}{l}1 \\ 1\end{array}\right]$. The dashed curve denotes the quark contribution which is slightly increasing towards large values of $x_{\gamma}$. In contrast, a sizable gluon contribution develops in the $q \bar{q}$ state of the photon at small values of $x_{\gamma}$.

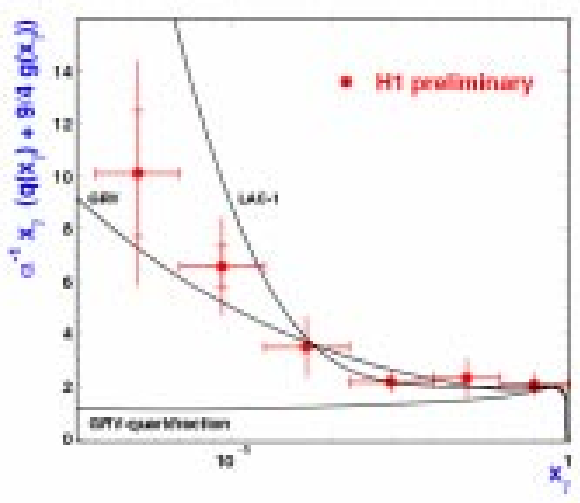

Figure 13: Combined probability of finding a quark or gluon in the photon with fractional momentum $x_{\gamma}$. The quark fraction is constrained by measurements of the photon structure function $F_{2}^{\gamma}$ at electronpositron colliders. The HERA data show the gluon distribution of the photon for the first time. The curves reflect different parameterizations of the photon constituents based on the $F_{2}^{\gamma}$ measurements.

\section{The Structure of Colour Singlet Exchange}

The cross sections of elastic scattering between hadrons cannot be explained by the exchange of a single boson (photon, Z, W, gluon). Instead the interaction can be viewed as being mediated by a colour singlet state $\mathbb{P}$ (Pomeron) which is composed of quarks and gluons (figure 14

The structure of this state was analysed at HERA, using for the first time deep inelastic scattering processes with electrons. Signature for such processes is the simultaneous detection of the scattered electron and the scattered proton. Owing to the lack of statistics, an alternative 
method is used in addition where instead of detecting the scattered proton a phase space region in the proton direction is demanded to be free of hadrons (figure $\left.\underline{1}^{1} \overline{5}_{1}^{i}\right)$.

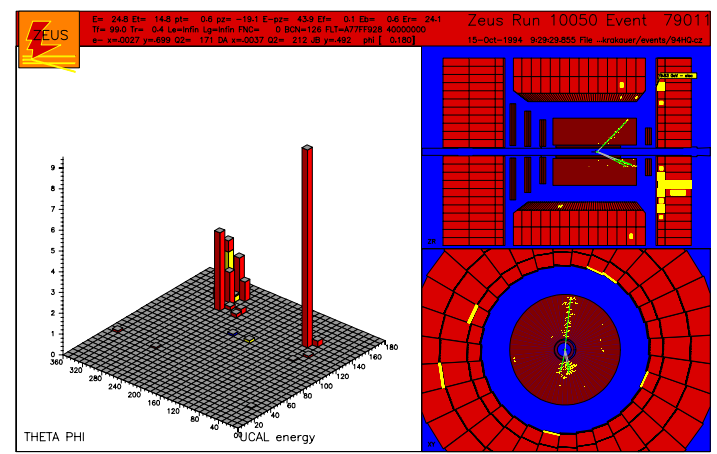

Figure 15: Deep inelastic electron-proton scattering with photon and colour singlet exchange: the upper right display gives the side view of the Zeus detector where the electron enters from the left and the proton from the right. The electron is visible pointing to the right upper half. The jet pointing to the lower right direction reflects the scattered quark. In contrast to the event displays shown so far, no hadron is visible in the direction of the outgoing proton which is a signal for colour singlet exchange from the proton. The lower right part of the display gives the view along the beam line showing that the electron and jet are "back-to-back". The left histogram shows the distributed energies in the phase space of rapidity and azimuthal angle.

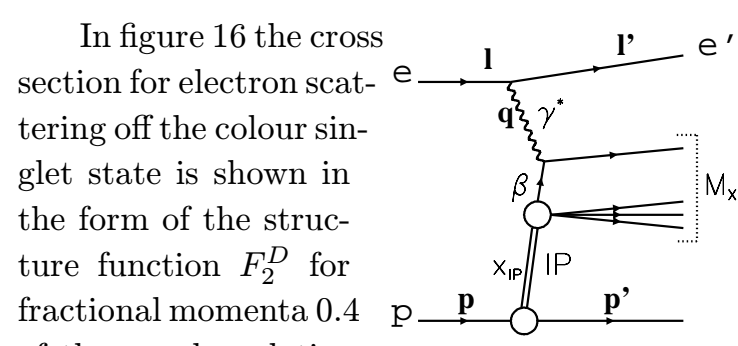

of the quarks relative

to the colour singlet stateFigure 14: Deep in$\mathbb{P}[\overline{6} \mid$. The fundamen- elastic electron-Pomeron tal QCD evolution equa- scattering with photon tion ' 2.10 provides the exchange.

key to understanding

this measurement: the structure function of the colour singlet exchange is found to be more or less independent of the resolution power $Q^{2}$ of the scattering processes. In contrast, the structure function of the proton falls at this large value of $x=0.4$ (figure $\left.{ }_{1}^{-1} \overline{1} \overline{1}\right)$, since the number of constituents in the proton well above the average valence quark fractional momenta $x \sim 0.1$ is small. Therefore, the colour singlet state is not a hadron with valence quarks as the proton. Instead its structure is at large values of the fractional momenta dominated by gluons which split into quark-antiquark pairs to enable the process of electron-quark scattering.

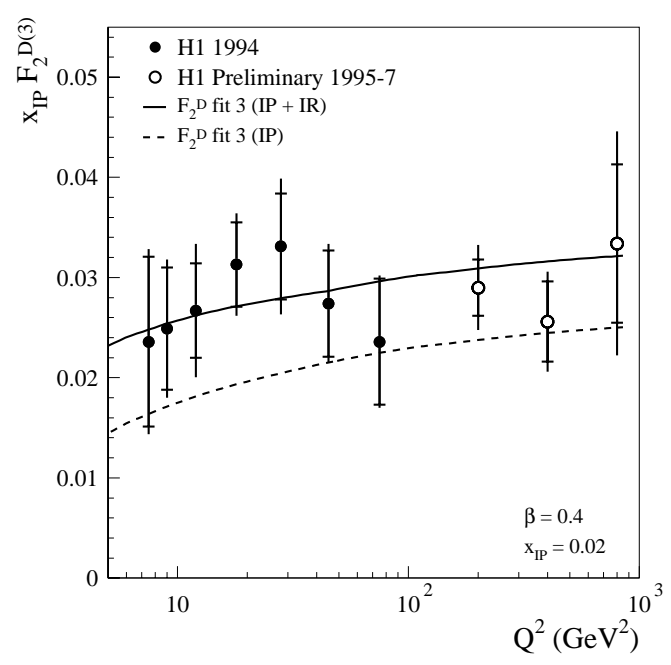

Figure 16: The structure function $F_{2}^{D}$ of colour singlet exchange as a function of the resolution power $Q^{2}$ for fractional momentum 0.4 of the scattered quark relative to the colour singlet state. The curves show the results of a QCD fit to many data points.

The measurements on the structure of colour singlet exchange have renewed strong theoretical efforts in trying to understand such processes from fundamental principles.

\section{Observation of Unusual Event Sig- natures}

With great care the lepton-proton data are checked for compatibility with the Standard Model. Two signatures were observed with more events measured than expected:

\subsection{Events at High $Q^{2}$}

In deep inelastic electron-proton scattering at very large scattering angles, i.e. large resolution power $Q^{2}>15000 \mathrm{GeV}^{2}$, the experiments $\mathrm{H} 1$ and Zeus observed more events than expected (figure '1" 


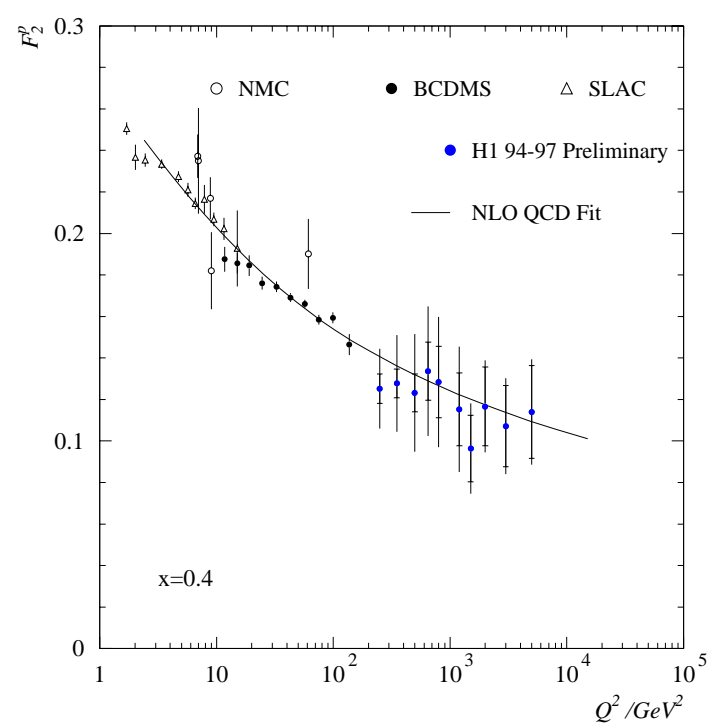

Figure 17: Proton structure function as a function of the resolution power $Q^{2}$ at quark fractional momentum $x=0.4$. The curve shows a QCD fit to many data points.

[1] events is an invariant mass of the electron-quark systems close to $200 \mathrm{GeV}$ (figure $1 \overline{1} \overline{1}$ ) which has caused major discussions and theoretical work. Such signatures are expected for the formation of new particles, for example a bound state of an electron and a quark, so called leptoquarks. In comparison with other colliders, the sensitivity to leptoquarks in lepton-proton interactions is superior in the region of small branching ratios of the leptoquark decays: the discovery potential at HERA is unique.

However, the statistical significance of the observed excess is at the level of 2 standard deviations. A verification of this observation in the high statistics data taking after the upgrade of HERA is therefore mandatory.

\subsection{Events with Isolated Muons and Miss- ing Transverse Momentum}

With the $\mathrm{H} 1$ experiment 5 events were found with a highly energetic muon and a hadron jet which are well separated from each other. In addition large missing transverse momentum was

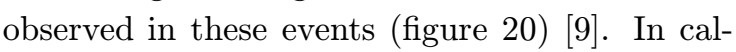
culations using the Standard Model only 1 event with this signature is expected from $\mathrm{W}$ boson
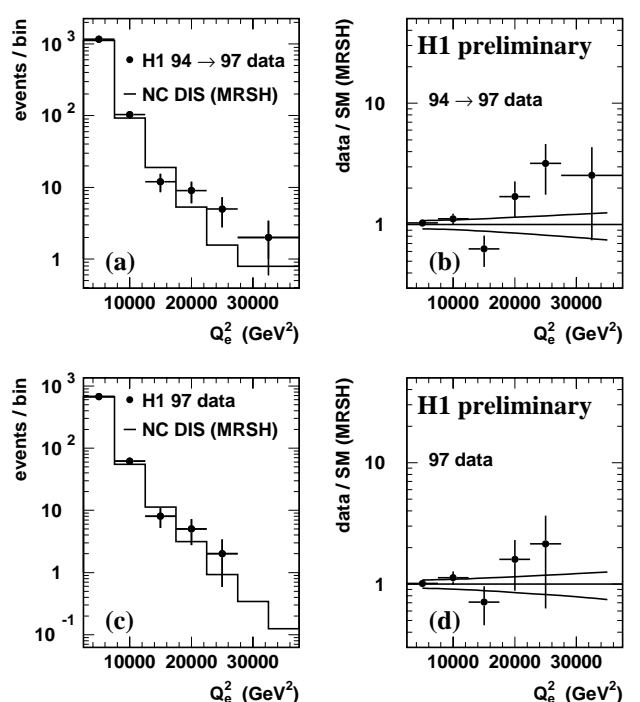

Figure 18: Measured event yields in positronproton scattering at large resolution power $Q^{2}$ for neutral and charge exchange processes in comparison with Standard Model expectations. An excess of the data relative to the Standard Model calculation is visible at high $Q^{2}$.

production where the $\mathrm{W}$ decays into a muon and a neutrino. A possible explanation for the observed signature is the production of the Super Symmetric partner particle of the top quark, the so called stop quark. Also for these events data taking with high statistics is mandatory and the confirmation of the Zeus experiment is needed.

\section{Conclusion}

The enthusiasm for the physics at HERA now and for the coming high luminosity upgrade program results from

- the large variety of measurements which substantially improve our understanding of particle constituents and their dynamics: proton, photon, mesons, colour singlet exchange, and

- the interesting high- $Q^{2}$ and muon events recorded which potentially are indicators for new particles or new phenomena. 


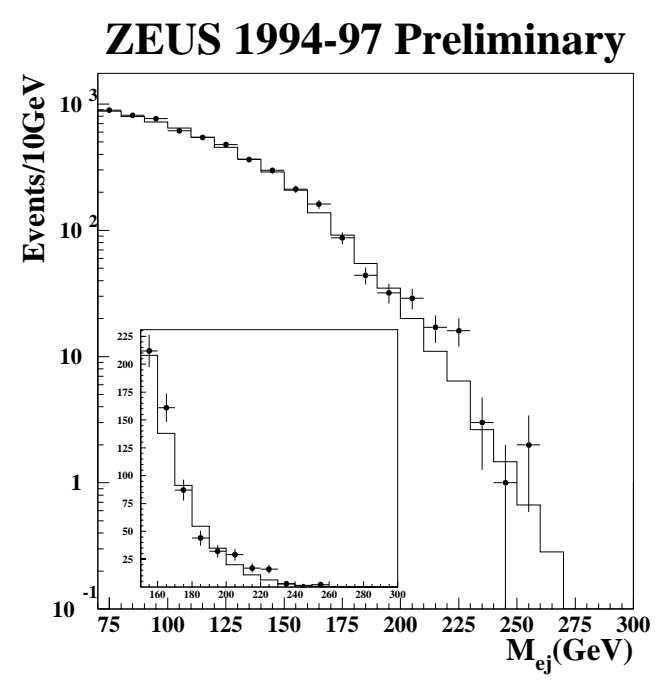

Figure 19: Measured event yields in positronproton scattering as a function of the invariant scattered-positron-jet mass. An excess of events is visible relative to the Standard Model calculation (histograms) around $M_{e j} \sim 200 \mathrm{GeV}$.

\section{Acknowledgments}

I wish to thank the organizers very much for the interesting combination of school and workshop in an environment that is exceptionally beautiful! E.Elsen I wish to thank very much for carefully reading the manuscript.

\section{References}

[1] A.T. Doyle, "Structure Functions", Talk given at the 29th Intern. Conf. on HighEnergy Physics, Vancouver, Canada (1998) hep-ex/9812029.; H1 Collab., "Precision Measurement of the Inclusive Deep Inelastic ep Scattering Cross Section at Low $Q^{2}$ at HERA" Conf. Paper 534; ZEUS Collab., "Measurement of the Proton Structure Function $F_{2}$ in $e^{+} p$ Collisions at HERA" Conf. Paper 769.

[2] H1 Collab., "Measurement of Inclusive Cross Sections for Neutral and Charged Current Interactions at High- $Q^{2}$ ", Conf. Paper 533, 29th Intern. Conf. on High-Energy Physics, Vancouver, Canada (1998).

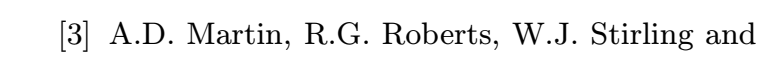

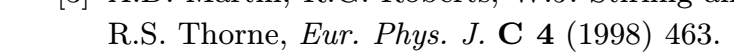

[4] ZEUS Collab., "High-Mass Di-Jet Cross Sections in Photoproduction at HERA", Conf. pa-

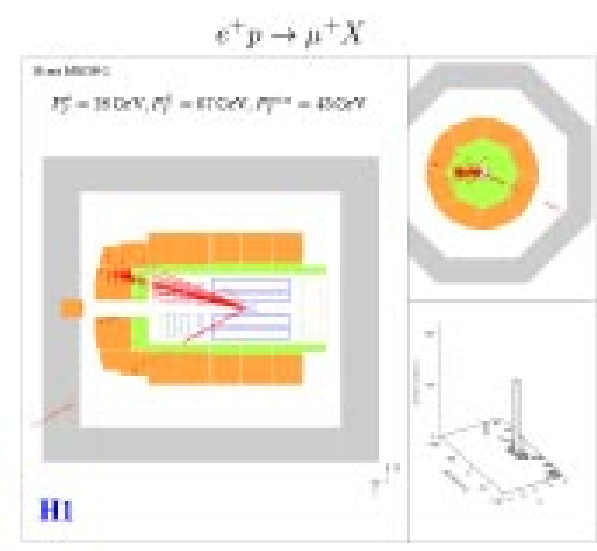

Figure 20: Muon event: the main display (left) gives the side view of the $\mathrm{H} 1$ detector where the electron enters from the left and the proton from the right. The muon points to the lower left direction and penetrates the iron yoke. A jet points to the upper left direction. The upper right part of the display gives the view along the beam line showing that the muon and the jet are not balanced which signals missing transverse momentum. The lower right histogram shows the distributed energies in the phase space of rapidity and azimuthal angle.

per 805, 29th Intern. Conf. on High-Energy Physics, Vancouver, Canada (1998).

[5] J.Cvach for the H1 Collab., "Real and Virtual photon structure from dijet events", Talk given at the 7th International Workshop on Deep Inelastic Scattering and QCD, Zeuthen, Germany (1999).

[6] H1 Collab., "Measurement and Interpretation of the Diffractive Structure Function $F_{2}^{D(3)}$ at HERA", Conf. Paper 571, 29th Intern. Conf. on High-Energy Physics, Vancouver, Canada (1998).

[7] H1 Collab;, C.Adloff et al., 'ZZ. Physik $\mathbf{C} \mathbf{7} \overline{\mathbf{7}}$ (1997) 191;; "A Search for Leptoquark Bosons in DIS at High $Q^{2}$ at HERA", Conf. Paper 579, 29th Intern. Conf. on High-Energy Physics, Vancouver, Canada (1998). 
[8] ZEUS Collaboration; J.Breitweg et al. ' $\bar{Z}$.' '- - _ Physik C 74 (1997) 207; "Search for Narrow High Mass States in Positron-Proton Scattering at HERA", Conf. Paper 754, 29th Intern. Conf. on High-Energy Physics, Vancouver, Canada (1998).

[9] H1 Collab., C. Adloff et al., Eur. Phys. J. C 5 (1998) 575. 